\title{
Two or More Persisting New Bone Lesions Indicator
}

National Cancer Institute

\section{Source}

National Cancer Institute. Two or More Persisting New Bone Lesions Indicator. NCI

Thesaurus. Code C132465.

An indication as to whether there are two or more bone tumors that have been

previously seen or characterized. 